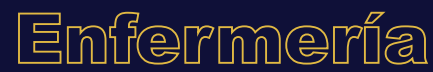

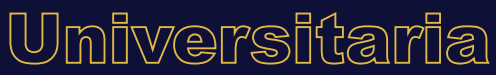

\section{A un año de gestión en la Escuela Nacional de Enfermería y Obstetricia, iniciando el reto: la transformación}

\section{One year in the Escuela Nacional de Enfermería y Obstetricia management, the beginning of the challenge: the transformation}

\author{
Há um ano da gestão na Escola Nacional \\ de Enfermagem e Obstetrícia, iniciando \\ o desafio: a transformação
}

La Enfermería es hoy en la UNAM una de las carreras de alta demanda. Sirvan estos datos para ejemplificarlo: cada año casi 14 mil jóvenes solicitan ingresar a la Licenciatura en Enfermería; se cuenta con 1340 lugares, insuficientes para cubrir la demanda, por lo que la matrícula se amplía para aceptar a 1600 estudiantes; la mitad ingresará a la Escuela Nacional de Enfermería y Obstetricia (ENEO). Esta situación nos obliga a redoblar esfuerzos para responder con calidad educativa a la sociedad que, ve en la Enfermería una excelente opción de vida profesional.

La ENEO es una institución en pleno crecimiento, que tiene un fuerte compromiso social con la nación para formar a profesionales en dos licenciaturas, tanto en la modalidad escolarizada como en línea; ofrece 15 especialidades de posgrado, una maestría en enfermería, un doctorado en proceso de aprobación; así como diversos programas de educación continua. Todos ellos acreditados por los organismos nacionales correspondientes.

A punto de informar a la Comunidad, las Autoridades Universitarias y al Honorable Consejo Técnico de la ENEO sobre los resultados obtenidos en este año de gestión, se exponen los avances más relevantes y logros obtenidos en el año 2019 correspondientes a: 7 programas estratégicos, 37 proyectos, 25 subproyectos, 240 metas y 342 indicadores que conforman el Plan de Desarrollo Institucional 2019-2023 acordes a las funciones sustantivas de la UNAM (Docencia, Investigación, Extensión y Difusión de la Cultura, así como Vinculación y Gestión). Tareas indispensables para el avance de los diversos procesos que subyacen a la formación de profesionales, especialistas y maestros en enfermería y de los diferentes programas que le acompañan. 
En este primer informe de actividades, es posible apreciar la labor realizada para lograr los objetivos institucionales de nuestra Escuela, siempre a la búsqueda de calidad en la formación universitaria de enfermeras y enfermeros. Es producto de la suma de esfuerzos de la comunidad estudiantil, cuerpo académico, personal administrativo de base, empleados de confianza y equipo de gestión responsable de cada área, los cuales desarrollan sus funciones, a fin de cumplir con los compromisos planteados ante la honorable Junta de Gobierno y con la comunidad universitaria en su conjunto.

La ENEO tiene como misión formar profesionales con altos estándares de calidad, para dar respuesta a las exigencias de cuidado de las personas en sus diferentes etapas de vida, al igual que impulsar la creación y difusión de nuevo conocimiento a través de la investigación en los campos de salud y en la formación de recursos humanos. Somos un espacio donde se discute, construye y recrea el saber necesario para resolver problemáticas de la atención a la salud de la población, además de tener el compromiso de coadyuvar en el cuidado integral de la sociedad, de manera humana, profesional, responsable y ética.

La ENEO es, sin duda, un lugar excepcional para estudiar enfermería en nuestro país; forma parte de la UNAM, universidad ubicada en el primer lugar del ranking OS en el área de Ciencias de la Salud en Iberoamérica, lo que nos compromete a ser una institución de calidad. Atendemos a una población estudiantil de más de cinco mil alumnos, con una plantilla académica mayor a 550 docentes, muchos de los cuales tienen estudios de posgrado, y cerca de una decena de ellos pertenece al Sistema Nacional de Investigadores del Consejo Nacional de Ciencia y Tecnología CONACyT. Asimismo, un gran porcentaje de nuestro profesorado trabaja en el Sistema Nacional de Salud, lo que les permite vincular teoría y práctica. Ellas y ellos son el motor de nuestra institución. Su ubicación geográfica al sur de la Ciudad de México le permite mantener vinculación estrecha con las dependencias del sistema de salud federal y local que se ubican en esta zona.

Para dar cobertura a toda la población estudiantil, disponemos de espacios académicos de posgrado en Ciudad Universitaria; la División de Educación Continua en Mixcoac; y el Centro Universitario de Enfermería Comunitaria en Xochimilco, a través del Sistema de Universidad Abierta y Educación a Distancia (SUAyED). Estos espacios cuentan con instalaciones modernas y tecnología para ofertar servicios educativos de máxima calidad.

En su carácter nacional, la Escuela tiene sedes y subsedes en toda la República Mexicana, donde se imparte la Licenciatura en Enfermería, además de Posgrados del Programa Único de Especialización en Enfermería. Con esta infraestructura, ofrecemos formación profesional y especializada presencial, semipresencial y a distancia. Lo anterior, nos permite ser un pilar fundamental para la profesionalización de los recursos humanos en el sistema de salud.

Nuestras licenciaturas se caracterizan por mantener un alto nivel educativo, gracias a la constante revisión de planes y programas de estudio, a la formación y desarrollo de la planta académica (profesores, tutores y técnicos académicos). Especial atención merece, en la Escuela, la formación integral que se oferta a nuestros estudiantes, quienes cuentan con un plan flexible, opciones de movilidad nacional e internacional y aprendizaje del idioma inglés; igualmente tienen diversas modalidades para la titulación, con opción de elegir aquella que mejor se adapte a sus intereses y posibilidades. Aunado a esta educación integral, contamos con espacios totalmente equipados para realizar actividades académicas, culturales, deportivas y administrativas, tales como servicio médico, comedor, biblioteca, gimnasio al aire libre y techado, mediateca, laboratorios de cómputo y laboratorios móviles PC-Puma. En ellos, el estudiantado tiene espacios óptimos y condiciones favorables 
para la práctica de hábitos saludables. También se fomenta la creatividad, a través de la posibilidad de experimentar diversas disciplinas artísticas como danza, música, teatro, cine y artes en general. En los últimos años, los estudiantes de la escuela destacaron en disciplinas deportivas como judo, baloncesto, voleibol de playa y hockey sobre pasto, en las que obtuvieron premios y reconocimientos. Además del deporte de competencia, se promueve la activación física, tanto en instalaciones deportivas como en espacios laborales, a través del programa Pausa para la Salud, en el que participan trabajadores administrativos, profesorado y estudiantes. Es así como la ENEO contribuye a la formación integral, sustentada en rigurosos principios científicos-humanísticos.

La enseñanza práctica de la enfermería es hoy, más que nunca, un reto, por la velocidad con la que se mueve el conocimiento. Para afrontar este panorama, la ENEO dispone de los Centros de Enseñanza Clínica Avanzada e Interactivo de Morfofisiología, los cuales ofrecen a la comunidad estudiantil la oportunidad de adquirir habilidades clínicas para responder a situaciones reales, gracias a simuladores, modelos y tecnología de última generación. Ahí los estudiantes practican en escenarios virtuales, de forma segura, guiados por profesores y tutores del más alto nivel. La ENEO cuenta también con un quirófano, un Centro para la Enseñanza de la Enfermería Obstétrica y Neonatal, un Laboratorio de Farmacología Clínica, Ciencias Biológicas y de la Salud, y un Laboratorio para el Cuidado Holístico, donde el papel de los profesores, técnicos académicos y tutores es fundamental para conducir el proceso enseñanza-aprendizaje. Por todo ello, ambas licenciaturas se encuentran re-acreditadas ante los organismos nacionales: el Consejo Mexicano para la Acreditación de la Enfermería (COMACE) y la Comisión Interinstitucional para la Formación de Recursos Humanos para la Salud (CIFRHS).

La ENEO tiene la oferta educativa de especialización de posgrado en enfermería más diversa y consolidada del país, por lo que se encuentra en el Programa Nacional Posgrados de Calidad del CONACyT. Las 15 especializaciones con las que contamos, permiten al profesional de enfermería profundizar sus conocimientos en áreas como salud pública, perinatal, infantil, cardiovascular, anciano, cultura física y deporte, neurología, salud mental, perioperatorio, rehabilitación, atención en el hogar, entre otras. Todas responden adecuadamente a las necesidades de la sociedad mexicana, e incluso, se anticipan a las perspectivas del rol ampliado de la atención de enfermería, que la población requerirá en los próximos años. También participamos en el programa de Maestría en Enfermería, el cual busca formar profesionistas del más alto nivel con capacidad para desempeñar distintos roles: docente, líder y gestor, así como para el trabajo de investigación pues lo inicia en la publicación y generación de conocimientos. Dicho programa está acreditado por el CONACyT en el nivel consolidado, y se encuentra en proceso de obtener indicadores de competencia internacional.

La ENEO aspira en los próximos años a cumplir requisitos universitarios para transitar a Facultad. Por ello, participa activamente en el diseño del plan de estudios del programa de Doctorado en Enfermería, la formación de la planta docente y consolidación de la investigación; el programa se encuentra en proceso de aprobación por los organismos colegiados universitarios.

Para cumplir con la actividad sustantiva de investigación en la ENEO, participan profesores de carrera y de asignatura, técnicos académicos, alumnos de servicio social y de posgrado. Nuestros investigadores, estudiantes y profesorado, asisten a conferencias tanto en México como en otros países; publican en revistas indizadas nacionales e internacionales, y participan en estancias de investigación en otros centros de estudio. Conformados en tres grandes Líneas de Generación y Aplicación del Conocimiento, sus proyectos cuentan con financiamiento de la ENEO, de los pro gramas universitarios PAPIIT y PAPIIME, así como de instituciones de asistencia privada como la 
Fundación Río Arronte. Para la difusión científica, se cuenta con la mejor revista de enfermería del país y una de las mejores de la región; la revista Enfermería Universitaria es de acceso abierto y está disponible en tres idiomas (español, inglés y portugués), con lo cual refrendamos nuestro compromiso de producir y difundir el conocimiento. Está incluida en las bases de datos más importantes de Iberoamérica: BVS, CUIDEN, SCIELO, Redalyc, Dialnet, DOAJ y AmeliCA, lo que la convierte en una de las publicaciones científicas de enfermería más importantes; además, forma parte del catálogo de 200 Universidades alrededor del mundo.

Nuestra escuela mantiene vínculos estrechos con los sectores público y privado, así como con la sociedad en general, a través de convenios de colaboración que permiten brindar servicios educativos diversos de educación continua y extensión universitaria. De igual manera, en colaboración con otras dependencias del gobierno local, realizamos Ferias de la salud, campañas y voluntariado, en una multiplicidad de escenarios comunitarios, centros de salud y hospitales. Un ejemplo destacado de este vínculo es el Centro Universitario de Enfermería Comunitaria, localizado en la Alcaldía de Xochimilco, el cual atiende a personas en situación vulnerable con servicios de prevención y promoción de la salud. Dicho Centro permite el aprendizaje y ejercicio del rol ampliado de enfermería en el primer nivel de atención, el trabajo interprofesional, el fortalecimiento de los vínculos con la comunidad y la visibilidad de la enfermería en la comunidad por su capacidad resolutiva, además de su gran sentido profesional y humano.

Este año, la escuela ha intensificado su participación como Centro Colaborador de la Organización Mundial de la Salud y de la Organización Panamericana de la Salud para el Desarrollo de la Enfermería Profesional; la ENEO en abril fue redesignada por cuatro años para realizar cooperación técnica en el país, en la formación de recursos humanos, la profesionalización y el impulso de la práctica avanzada de la enfermería. Como tal, se unió a la campaña Nursing Now, para apoyar el liderazgo de la disciplina en el mundo entero y participa en el Año Internacional de la Enfermería 2020, impulsado por el Consejo Internacional de Enfermería y, en México, por la Dirección de Enfermería de la Secretaría de Salud.

Para apoyar a la comunidad estudiantil, contamos con programas de becas de manutención, investigación, deportiva, de titulación; idiomas, servicio social, así como movilidad e intercambio académico. En este último rubro, nuestros estudiantes y el personal académico tienen la posibilidad de realizar estancias de movilidad, tanto en México como en el extranjero, a través de convenios y con el apoyo de la Dirección General de Cooperación Internacional.

Para finalizar, la ENEO, con más de un siglo de historia, es hoy una escuela en constante desarrollo y transformación, reconocida por contar con excelente profesorado, planes de estudio de vanguardia, recursos tecnológicos para la enseñanza de la disciplina y por haber formado a un gran número de líderes en el gremio. Se mantiene así, como la mejor oferta educativa para el estudio y la generación del conocimiento de la enfermería como disciplina y profesión del cuidado de la salud humana.

Rosa A. Zárate-Grajales

ORCID: https://orcid.org/0000-0002-9264-8490

Directora de la Escuela Nacional de Enfermería y Obstetricia, Universidad Nacional Autónoma de México Ciudad de México, México r.zarate@comunidad.unam.mx 